\section{Fragmentary progress in endometriosis}

\section{By Michael J. Haas, Senior Writer}

A team from the Baylor College of Medicine has treated endometriosis in mice by blocking a pathway that produces a fragment of nuclear receptor coactivator 1 in endometrial tissue. ${ }^{1}$ Now, small molecule inhibitors are needed to elucidate the fragment's precise role in endometriosis and determine whether other pathway components are potential disease targets as well.

Endometriosis affects about $10 \%$ of women of reproductive age and involves the ectopic growth of tissue from the uterine lining (endometrium) in the peritoneal cavity, resulting in pelvic pain, infertility and other symptoms. The underlying pathogenesis of the disease is not well understood. What is known is that endometriotic lesions produce high levels of estrogen that enable growth of the lesion.

As a result most therapies reduce levels of estradiol and other estrogens. Treatments include GnRH/LHRH receptor-targeting compounds, aromatase inhibitors or contraceptives that can only halt-not reverse-lesion growth and may not completely control pain. Additionally, these therapies can have side effects such as cognitive deficits, hirsutism (excessive hairiness), the inability to conceive and an increased risk of osteoporosis.

Previous studies have shown that levels of nuclear receptor coactivator 1 (NCOA1; SRC1) were lower in patients' endometriotic tissue than in their normal endometrial tissue. However, the studies did not investigate whether the protein actually played a role in the disease. $^{2,3}$

Thus, the Baylor College of Medicine team decided to take a closer look at whether SRC1 might be a therapeutic target to treat endometriosis.

The group began by confirming that endometriotic tissues from patients and mouse models of the indication had lower levels of intact $(160 \mathrm{kDa}) \mathrm{SRC} 1$ than normal endometrial tissues. In doing so, the team found endometriotic tissue from patients and models had higher levels of a $70 \mathrm{kDa}, \mathrm{C}$-terminal fragment of SRC1.

In human endometrial cell lines, overexpression of the SRC1 fragment induced greater invasiveness than overexpression of fulllength SRC1 by promoting epithelial-mesenchymal transition (EMT). The fragment also enabled the cell lines to evade normal caspasemediated apoptosis that is triggered by the proinflammatory cytokine tumor necrosis factor- $\alpha$ (TNF- $\alpha$ ) and occurs in endometrial cells during menstruation.
Additional studies in endometrial and endometriotic tissue from the mouse models showed that Tnf- $\alpha$ upregulated matrix metalloproteinase 9 (Mmp9), which in turn cleaved full-length $\mathrm{Src} 1$ to produce the fragment (see Figure 1, "SRC1 fragments on the endometriotic pathway").

Lastly, the team showed that mice deficient in Tnf- $\alpha, M m p 9$ or $S r c 1$ had endometriotic lesions that were up to five times smaller than lesions in wild-type control models.

In its report in Nature Medicine, ${ }^{1}$ the team noted that the role of the TNF- $\alpha$-MMP9-SRC1 fragment pathway in endometriosis agreed with a host of previous studies. These included papers that described an association between high peritoneal levels of TNF- $\alpha$ and endometriosis progression in patients, ${ }^{4}$ showed a treatment effect for anti-TNF- $\alpha$ antibodies in rat $^{5}$ and baboon ${ }^{6}$ models of the disease, and found higher levels of MMPs in invasive endometriotic cells than in normal endometrial cells from patients and chicken models. ${ }^{7,8}$

\section{Trial and trial again?}

Of the three proteins in the pathway, the SRC1 fragment is the logical therapeutic target, team leader Bert O'Malley told SciBX.

"Drugs that inhibit TNF- $\alpha$ are already available, but their immunosuppressive activity has severe side effects, such as increased risk of pneumonia, tuberculosis and-worst of all-cancer, while inhibiting MMP9 can also have musculoskeletal and many other side effects," he said.

O'Malley is professor and chair of molecular and cellular biology at the Baylor College of Medicine.

Other endometriosis researchers agreed that the potential side effects of MMP9 inhibition ruled out the proteinase as an endometriosis target. Opinions were mixed on whether TNF- $\alpha$ or the SRC1 fragment will be the better therapeutic target.

Thomas D'Hooghe, coordinator of the Leuven University Fertility Center at University Hospitals Leuven and leader of the baboon model study, said the findings in the new paper actually bolster the case for TNF- $\alpha$ as a therapeutic target in endometriosis. "TNF- $\alpha$ inhibitors should be explored in a relevant clinical study, since all nonhuman primate studies suggest a treatment effect for them," he said.

D'Hooghe is also professor of medicine at Catholic University Leuven and adjunct professor of medicine at Yale University.

Thomas Collet, president and CEO of Meditrina Pharmaceuticals
"TNF- $\alpha$ inhibitors should be explored in a relevant clinical study, since all nonhuman primate studies suggest a treatment effect for them."

-Thomas D'Hooghe, University Hospitals Leuven

\section{Inc., cautioned that anti-TNF- $\alpha$}

therapy already has failed in at least one clinical trial to treat the disease.

In 2008, researchers from University Hospitals Leuven, the University of Oxford and Johnson \& Johnson's Centocor Inc. unit reported that Remicade infliximab, the pharma's chimeric $\mathrm{mAb}$ against TNF- $\alpha$, failed to reduce pain and lesion growth in a Phase II 
Four TNF- $\alpha$ inhibitors are marketed to treat rheumatoid arthritis (RA), Crohn's disease, inflammatory bowel disease (IBD), psoriasis, ankylosing spondylitis and/or other autoimmune diseases: Humira adulimumab, a human mAb against TNF- $\alpha$ from Abbott Laboratories (NYSE:ABT) and Eisai Co. Ltd. (Tokyo:4523; Osaka:4523); Enbrel etanercept, a recombinant p75 TNF receptor linked to the Fc portion of human IgG1 (TNFr:Fc) from Amgen Inc. (NASDAQ:AMGN), Pfizer Inc. (NYSE:PFE) and Takeda Pharmaceutical Co. Ltd. (Tokyo:4502); Remicade infliximab, a chimeric mAb against TNF- $\alpha$ from Johnson \& Johnson (NYSE:JNJ), Merck \& Co. Inc. (NYSE:MRK) and Mitisubishi Tanabe Pharma Corp. (Tokyo:4508; Osaka:4508); and Cimzia certolizumab pegol, a pegylated humanized antibody fragment against TNF- $\alpha$ from UCB Group (Euronext:UBC) and Astellas Pharma Inc. (Tokyo:4503).

Additionally, Johnson \& Johnson markets Remicade to treat Behçet's disease and spinal cord injury (SCl); and Marnac Inc., Ildong Pharmaceutical Co. Ltd., InterMune Inc. (NASDAQ:ITMN) and Shionogi \& Co. Ltd. (Tokyo:4507; Osaka:4507) market Esbriet pirfenidone, a small molecule inhibitor of proinflammatory cytokines such as TNF- $\alpha$ and profibrotic cytokines, to treat pulmonary fibrosis.

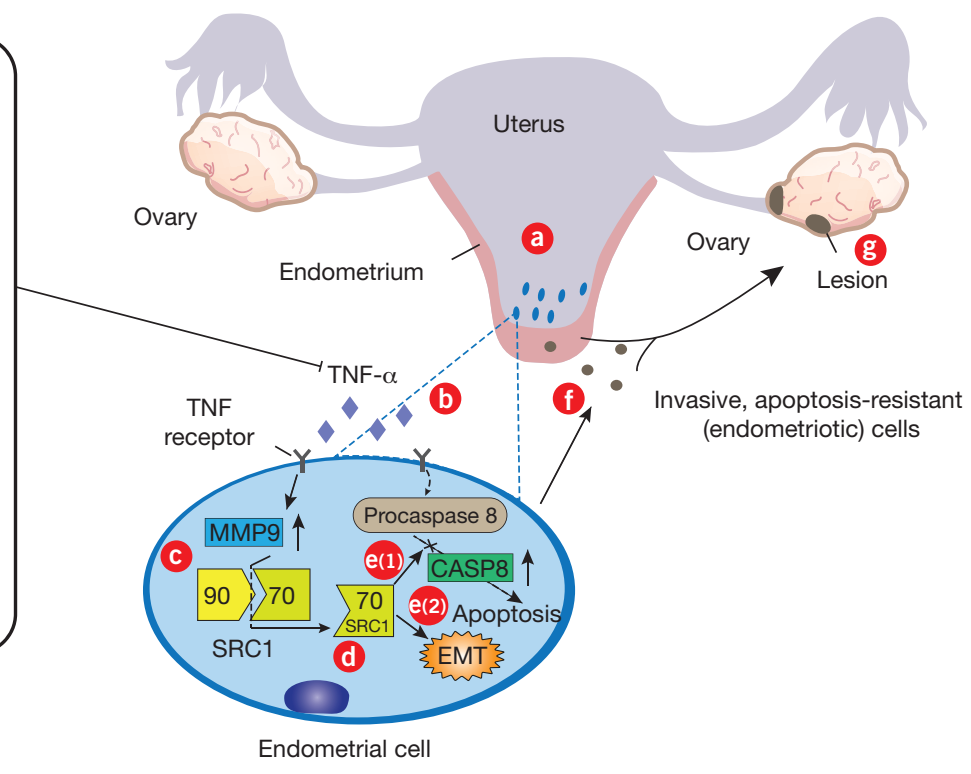

Figure 1. SRC1 fragments on the endometriotic pathway. According to a study in Nature Medicine, inhibition of a pathway that produces a fragment of nuclear receptor coactivator 1 (NCOA1; SRC1) in endometrial (uterine lining) cells could help treat endometriosis.

During menstruation, cells shed from the endometrium [a] can respond to local increases in tumor necrosis factor- $\alpha$ (TNF- $\alpha$ ) [b] by upregulating matrix metalloproteinase 9 (MMP9) [c], which cleaves a $70 \mathrm{kDa}$, C-terminal fragment from full-length (160 kDa) SRC1 [d]. In turn, the fragment prevents TNF- $\alpha$-induced activation of procaspase-8 to caspase-8 (CASP8; FLICE) [e(1)] and consequent apoptotic signaling, and it promotes the epithelial-mesenchymal transition (EMT) [e(2)] by an unknown mechanism, thereby inducing antiapoptotic and invasive phenotypes, respectively. These characteristics are the hallmarks of endometriotic cells [f] and enable them to form endometriotic lesions [g] on the ovaries and/or elsewhere in the peritoneum.

trial to treat endometriosis. ${ }^{9}$

D'Hooghe countered that patients in the infliximab trial "were those awaiting surgery for fibrotic, deeply invasive endometriotic nodules. We know that drug therapy has only limited success in such patients and that surgery is the best option for them." Thus, it was not surprising that infliximab failed to have an effect in this population, he said.

Instead, he wanted to see anti-TNF- $\alpha$ antibodies tested "in women with peritoneal endometriosis, who may have some small growths on their ovaries and a significant inflammatory phenotype" but less severe overall disease than the infliximab trial participants.

Eija Lundström, medical director at Debiopharm Group, agreed with O'Malley that TNF- $\alpha$ inhibitors were probably not ideal therapies for endometriosis.

Pamorelin LA triptorelin, a GnRH/LHRH receptor agonist from Debiopharm and Galenica Ltd.'s Vifor Pharma Ltd. unit, is approved to treat endometriosis, female infertility and advanced hormonedependent prostate cancer.

"Anything that could target the lesions locally without suppressing estradiol production would be advantageous" over current estrogensuppressing therapies, said James Symons, VP of clinical development at Meditrina.

Meditrina's MPI-676, an anastrozole-based aromatase inhibitor, is in Phase II testing to treat endometriosis.

J\&J, Merck \& Co. Inc. and Mitsubishi Tanabe Pharma Corp. market Remicade to treat rheumatoid arthritis (RA) and other autoimmune diseases. J\&J also markets the antibody to treat Behçet's disease and spinal cord injury (SCI).

If the SRC1 fragment is deemed a better target than TNF- $\alpha$, "it will be important to identify the intercellular partners of the SRC1 fragment" in addition to procaspase-8, said Michael Teifel, VP of preclinical development at Aeterna Zentaris Inc.'s Aeterna Zentaris $\mathrm{GmbH}$ unit. The reason, he said, is selective inhibition of the SRC1 fragment could block its antiapoptotic effects on endometrial cells but would not necessarily prevent it from promoting EMT.

Furthermore, the $S r c 1$ knockout models used by the team cannot distinguish between the fragment's role in disease onset and disease progression, said Joachim Fensterle, director of translational medicine at Aeterna Zentaris GmbH. "Validation of the pathway as a target for established disease would require additional in vivo experiments, such as a model in which $S r c 1$ could be conditionally knocked out or knocked down at different stages of disease," he said.

D'Hooghe added that he wants to see SRC1 fragment inhibitors tested in mouse and baboon models of endometriosis.

AEZS-115, a GnRH/LHRH receptor antagonist peptidomimetic from Aeterna Zentaris, is in preclinical development to treat endometriosis.

More broadly, Collet said any potential therapy to treat endometriosis has to account for the heterogeneity of the clinical presentation of the disease.

"For instance, while high estrogen levels are linked to disease progression, postmenopausal women can present with endometriosis" despite having lower estrogen levels than premenopausal women, which suggests several etiologies may contribute to disease onset or progression, he said. 
"Also, pain symptoms don't necessarily correlate with the size or the site of the lesion" but instead result from variability between individual patients and their pain experiences, Symons said.

"The key question is whether the findings reported in Nature Medicine could apply to all patients," Collet said.

O'Malley said his team has already conducted screens and identified inhibitors of the SRC1 fragment. The researchers plan to test the molecules in mice and potentially in monkey models.

His team also is considering testing the inhibitors in combination with existing therapies to look for potential additive or synergistic effects.

"It is possible that inhibitors of the pathway identified by the Nature Medicine team could have an additive or synergistic effect in combination with other therapies" to treat endometriosis, noted Lundström.

Longer term, the team wants to run prospective studies in patients to determine how broad a role the SRC1 fragment plays in endometriosis and to begin looking for specific links between that disease and cancer.

O’Malley said endometriotic cells undergo EMT and exhibit invasiveness similar to that seen in early stage cancers, and statistical studies have shown that endometriosis predisposes women to ovarian, uterine, colon, breast and other cancers.

The relationship between endometriosis and cancer is still poorly understood, he said.

If future studies uncover mechanistic links between the two diseases, "I think physicians will have to treat women who have endometriosis with more than just long-term therapies that address symptoms of pain," he said.

Baylor College of Medicine has applied for a patent covering the
SRC1 fragment inhibitors, and the findings are available for partnering or licensing, he said.

Haas, M.J. SciBX 5(26); doi:10.1038/scibx.2012.670

Published online June 28, 2012

\section{REFERENCES}

1. Han, S.J. et al. Nat. Med.; published online June 3, 2012; doi: $10.1038 / \mathrm{nm} .2826$

Contact: Bert W. O'Malley, Baylor College of Medicine, Houston, Texas e-mail: berto@bcm.tmc.edu

2. Suzuki, A. et al. Virchows Arch. 456, 433-441 (2010)

3. Kumagami, A. et al. J. Obstet. Gynaecol. Res. 37, 1269-1276 (2011)

4. Bedaiwy, M.A. et al. Hum. Reprod. 17, 426-431 (2002)

5. Islimye, M. et al. Eur. J. Obstet. Gynecol. Reprod. Biol. 159, 184-189 (2011)

6. Falconer, H. et al. Hum. Reprod. 21, 1856-1862 (2006)

7. Osteen, K.G. et al. Semin. Reprod. Med. 21, 155-164 (2003)

8. Nap, A.W. et al. Hum. Reprod. 19, 2180-2187 (2004)

9. Koninckx, P.R. et al. Hum. Reprod. 23, 2017-2023 (2008)

\section{COMPANIES AND INSTITUTIONS MENTIONED} Aeterna Zentaris Inc. (TSX:AEZ; NASDAQ:AEZS), Quebec City, Quebec, Canada

Baylor College of Medicine, Houston, Texas Catholic University Leuven, Leuven, Belgium Debiopharm Group, Lausanne, Switzerland Galenica Ltd. (SIX:GALN), Bern, Switzerland Johnson \& Johnson (NYSE:JNJ), New Brunswick, N.J. Meditrina Pharmaceuticals Inc., Ann Arbor, Mich. Merck \& Co. Inc. (NYSE:MRK), Whitehouse Station, N.J. Mitsubishi Tanabe Pharma Corp. (Tokyo:4508; Osaka:4508), Osaka, Japan

University Hospitals Leuven, Leuven, Belgium

University of Oxford, Oxford, U.K.

Yale University, New Haven, Conn. 